\title{
Walsh Transform Based Feature Vector Generation for Image Database Classification
}

\author{
Tanuja Sarode $^{1}$, Jagruti Save ${ }^{2}$ \\ ${ }^{1}$ Departement of Computer Engineering, Thadomal Shahani Engineering College, Mumbai \\ ${ }^{2}$ MPSTME, NMIMS University, Mumbai, India
}

\section{Article Info \\ Article history: \\ Received Oct 28, 2015 \\ Revised May 4, 2016 \\ Accepted May 20, 2016 \\ Keyword: \\ Feature Vector \\ Image Classification \\ Vector Quantization \\ Walsh Transform \\ Wavelet Transform}

\begin{abstract}
Thousands of images are generated everyday, which implies the need to build an easy, faster, automated classifier to classify and organize these images. Classification means selecting an appropriate class for a given image from a set of pre-defined classes. The main objective of this work is to explore feature vector generation using Walsh transform for classification. In the first method, we applied Walsh transform on the columns of an image to generate feature vectors. In second method, Walsh wavelet matrix is used for feature vector generation. In third method we proposed to apply vector quantization (VQ) on feature vectors generated by earlier methods. It gives better accuracy, fast computation and less storage space as compared with the earlier methods. Nearest neighbor and nearest mean classification algorithms are used to classify input test image. Image database used for the experimentation contains 2000 images. All these methods generate large number of outputs for single test image by considering four similarity measures, six sizes of feature vector, two ways of classification, four VQ techniques, three sizes of codebook, and five combinations of wavelet transform matrix generation. We observed improvement in accuracy from $63.22 \%$ to $74 \%$ (55\% training data) through the series of techniques.
\end{abstract}

Copyright ( 2016 Institute of Advanced Engineering and Science. All rights reserved.

\section{Corresponding Author:}

Tanuja Sarode,

Departement of Computer Engineering,

Thadomal Shahani Engineering Collage,

Mumbai, India.

Email:

\section{INTRODUCTION}

Recent increases in storage capacity, processing power, and display resolution have enabled large image database development. Due to advancement in internet technologies, these databases have tremendously grown further. The task of accessing, processing, analyzing, and sharing these images has become more difficult. If images are properly organized, then accessing these images will be fast. Hence, with the large availability of high quality digital images, the need of classifying/categorizing images automatically is becoming increasingly important and challenging nowadays. Human beings easily classify images even if the images are poorly illuminated, partially occluded, and noisy. However, the classification task is not easy for machine. Hence, to design generic image classifier remains an elusive goal. The term image classification is a process of assigning an image to one of the predefined class. Manual classification of relevant images from a large database is time consuming, laborious, expensive, and subjective. So many researchers have focused on automatic (machine) classification of images. Content-based image retrieval (CBIR) is a system of retrieving a set of images similar to query image from a large image database. A successful classification of images will greatly enhance the performance of CBIR system by filtering out images from irrelevant classes during matching [1]. The problem of classifying images in database into predefined category has many levels of generality [2]. It can be as broad as separating indoor and outdoor 
images [3] or different outdoor scenes [4], it can be as generic as separating car, flower, elephant classes, or it can be finer [5] as separating different types of cloud images [6]. In one class classification (Unary classification) problem, image of an object is classified as genuine object or an outlier object. This classification is useful in some data mining applications like outlier detection and anomaly detection [7],[8]. In two-class classification (binary classification) problem, test image is assign to one of the predefined two classes [9]. This method has an application in medical field to detect the abnormality in medical images [10]. If the classification is for more than two classes then we get multi class classification. Classification is also classified as supervised and unsupervised classification [11],[12]. In general, automatic supervised image classification contains two steps: 1. Feature Extraction 2. Build Classifier [13]. The simplest way to represent an image in fewer coefficients is to extract color, shape and texture information from an image and represent it in a compact form [14]-[17]. Global features are extracted from entire image whereas local features are extracted from parts of an image [18]. Feature extraction can be done in spatial domain or transform domain. Image transform such as Walsh Transform has a property of energy compaction. Maximum energy accumulated in fewer coefficients; hence, reduced feature vector size. Once the feature vector generated, classification procedures such as nearest neighbor classification [19], classification using artificial neural network [20], and support vector machine [21] build the classifier.

We proposed a system in which initially Walsh transform [22], applied to the columns of an image. Then row mean vectors for three planes combined to make feature vector of that image [23]. To make effective and compact representation of training set feature vectors, different techniques of vector quantization (VQ) are applied on training set. Nearest neighbor (NN) classifier and nearest mean (NM) classifier with different similarity measures [24] assign the output class for a test image. To enhance the accuracy of classifier model, second method suggests applying Walsh wavelet to the columns of an image instead of simple Walsh transform. Addition of VQ techniques increases accuracy further decreases time and storage required. The paper is organized as follows: Section 2 explains detailed procedure of proposed system. Section 3 discusses all the results of implementation. The conclusion is given in section 4 followed by references.

\section{RESEARCH METHOD}

The paper gives three different methods to generate training set of feature vectors as explained in section 2.1, 2.2, and 2.3.

\section{1. 'Walsh Transform over Row mean' based Feature vector Generation}

The stepwise procedure for the first method is as follows:

1. Apply Walsh Transform to the columns of three planes (R, G, and B) of training image.

2. Calculate the average of each row of transformed image planes. This will give one row mean vector of size 256x1 for each plane (size of each image 256x256) [25].

3. Organize first ' $Z$ ' values of these three vectors one below the other to generate feature vector of size ' $3 Z x 1$ '. By taking the value of 'Z' as 25, 50,100,150, 200 and 256, we get feature vector of different sizes such as $75 \times 1,150 \times 1,300 \times 1,450 \times 1,600 \times 1$ and 768x1 respectively. Above three steps are repeated for each training image. This will generate the training set.

4. Apply the above procedure for test image

5. Apply nearest neighbor (NN) classifier where all training feature vectors are used and apply nearest mean (NM) classifier where average feature vector of each class is used as training set. Figure 1 shows the procedures for these two classifiers. Different distance measures such as Euclidean distance, Manhattan distance, Cosine correlation similarity, and Bray-Curtis [26]-[28] are used to calculate distance between training feature vector and testing feature vector.

\subsection{Walsh Wavelet Transform based Feature vector Generation}

This method uses the procedure of generation of wavelet matrix from two orthogonal transform matrices. The algorithm is as follows:

1. Create Walsh wavelet matrix from two Walsh matrices [29]. To generate wavelet matrix of size 256x256, we can take two Walsh matrices of sizes '4x4 and $64 \times 64$ ' or ' $8 \times 8$ and 32x32' or ' $16 \times 16$ and 16x16'.

2. Apply steps 1 to step 5 from section 2.1 by replacing Walsh matrix with Walsh Wavelet matrix.

3. If wavelet matrix is created from two matrices of Walsh transform with sizes $8 \times 8$ and $32 \times 32$, and NN classifier is used for classification then the results are shown under the name Walsh_wavelet_8X32_NN and if NM classifier is used, the results are shown under the name Walsh_wavelet_8x32_NM.

4. Apply the above procedure for test image

5. Apply nearest neighbor (NN) classifier and nearest mean (NM) classifier. 

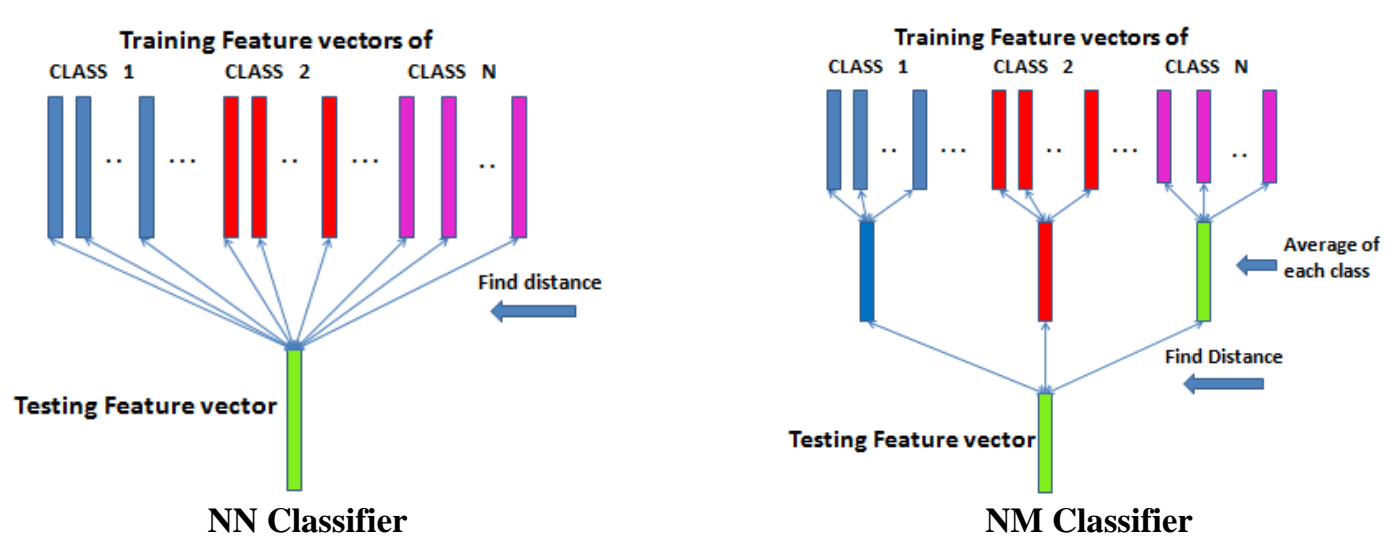

Figure 1. Nearest neighbour (NN) and nearest mean (NM) classifier

\subsection{Proposed method of Feature vector Generation}

The detailed procedure for method is as follows:

1. Apply first three steps from section 2.1 to generate training set.

2. Each feature vector of size 'Mx1' in a training set is a training point in 'M' dimensional space. Apply vector quantization techniques [30] such as Linde-Buzo-Gray (LBG) [31], Kekre's proportionate error (KPE) [32], Kekre's Fast Code book Generation (KFCG) [33] and Kekre's median codebook generation (KMCG) [34] algorithms to the training points of each class separately.

3. Generate the codebooks of size 4, 8 and 16. The code vectors are representative of the classes. Hence, the code vectors form the training set.

4. Apply NN classifier to find the class of test image.

The same procedure is applied on second method (Walsh wavelet based training set).

\section{RESULTS AND DISCUSSION}

For experimentation, large image database is constructed. This database contains total 2000 images (20 classes, 100 images per class). Six classes (bus, dinosaur, elephant, rose, horse, and mountain) are directly taken from Wang database [35]. Images of remaining fourteen classes (ibis bird, sunset, bonsai, car, panda, sunflower, airplane, coin, scooter, schooner, kingfisher bird, starfish, Windsor chair, and cup-saucer) are downloaded from the web related to the class keyword. Images are selected in such a way that they have many variations within the class and among the classes. Figure 2 shows the sample images of training database and testing database. Accuracy of classification is calculated as per equation (1). Initially 35 images per class are used for training purpose and remaining 65 images are used for testing purpose.Then training images increased by 10 for two times (45 images per class and then 55 images per class). It has been observed that as the number of training images increased accuracy increases. Table 1 indicates the size of training set, time required for generation of training set and time required for classification for single test image for different methods when we have used 35 images per class for training purpose. It shows that number of training feature vector reduces because of vector quantization hence reduces the classification time. Table 2 specifies highest accuracy obtained for all three methods for 55 training images and 45 testing images per class. Table 3 shows the variations in different factors considered for classification. Table 4 shows the confusion matrix obtained for 'Walsh_wavelet_32X8_NN' method (similarity criteria: Manhattan, number of training images: 55 images/class). This table shows the individual class performance.

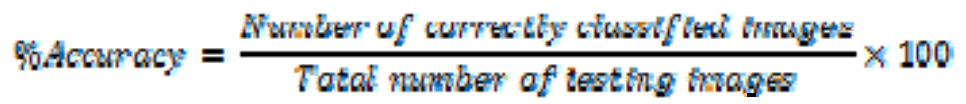

The sizes of codebook we have tried are 4, 8 and 16 because the minimum number of training images is 35 per class. It has been observed that the proposed method of VQ gives better results compared to earlier methods. 
Table 1. Number of training feature vectors and corresponding time required for each method for 35 training images/class

\begin{tabular}{|c|c|c|c|c|}
\hline \multirow[t]{2}{*}{ Method } & \multicolumn{2}{|c|}{$\begin{array}{l}\text { Number of training images : } 700 \\
\quad \text { (35 images/class) } \\
\text { Size of each feature vector : } 768 \times 1\end{array}$} & \multirow{2}{*}{$\begin{array}{l}\text { Time required } \\
\text { for generation } \\
\text { of all training } \\
\text { feature vectors } \\
\text { (in sec) }\end{array}$} & \multirow{2}{*}{$\begin{array}{c}\text { Time required } \\
\text { for classification } \\
\text { of single testing } \\
\text { feature vector } \\
\text { (in sec) }\end{array}$} \\
\hline & $\begin{array}{c}\text { No. of training } \\
\text { feature vectors/class }\end{array}$ & $\begin{array}{c}\text { Total training } \\
\text { feature vectors }\end{array}$ & & \\
\hline Walsh+Row mean+NN & 35 & 700 & 34.79 & 4.24 \\
\hline Walsh+Row mean+NM & 1 & 20 & 35.48 & 0.12 \\
\hline Walsh_wavelet+NN & 35 & 700 & 38.72 & 4.24 \\
\hline Walsh_wavelet+NM & 1 & 20 & 39.32 & 0.12 \\
\hline Proposed VQ(CB size 4) & 4 & 80 & 37.48 & 0.50 \\
\hline Proposed VQ(CB size 8) & 8 & 160 & 38.34 & 1.01 \\
\hline Proposed VQ(CB size 16) & 16 & 320 & 40.57 & 1.92 \\
\hline
\end{tabular}

Observations: 'NM' method requires least classification time and least storage space as the size of training set is only 20 training vectors. 'NN' method requires high classification time and large storage space as the size of training set is 700 training vectors. Proposed 'VQ' method generates training set of codebook (CB) size. It requires more classification time as compared to 'NM' method but it is quite less than 'NN' method.

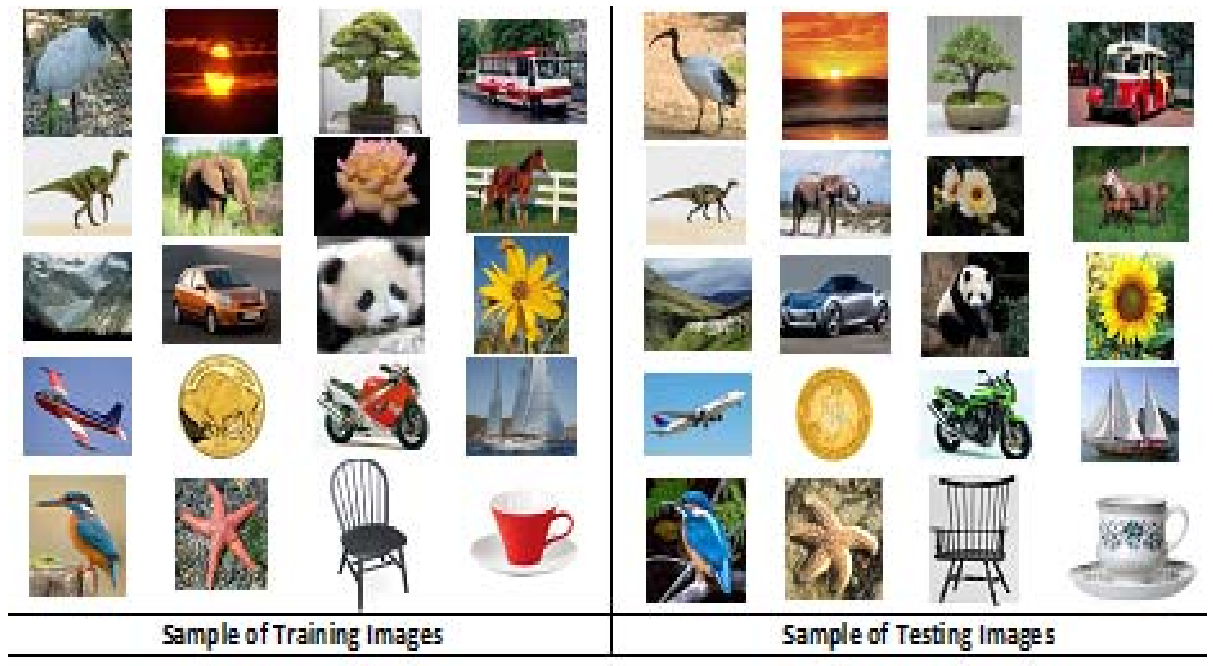

Figure 2. Sample images of training and testing Database

Table 2. Comparison of highest Overall Classification accuracy acheived for all the methods No. of training images: 1100 (55/class)

\begin{tabular}{ccccc} 
Method & $\begin{array}{c}\text { Highest \%accuracy } \\
\text { achieved }\end{array}$ & $\begin{array}{c}\text { Corresponding } \\
\text { feature vector size }\end{array}$ & $\begin{array}{c}\text { Corresponding } \\
\text { similarity criteria }\end{array}$ & VQ method/ Wavelet method \\
\hline Walsh+Row mean+NN & 61.22 & $600 \times 1$ & $\begin{array}{c}\text { Euclidean } \\
\text { Cosine correlation }\end{array}$ \\
Walsh+Row mean+NM & 52.67 & $300 \times 1$ & Manhattan & Walsh+Row mean+LBG \\
Proposed VQ(CB size 16) & $\mathbf{6 3 . 2 2}$ & $450 \times 1$ & Manhattan & Walsh_wavelet_32X8_NN \\
Walsh_wavelet+NN & 72.78 & $768 \times 1$ & Manhattan & Walsh_wavelet_32X8_NM \\
Walsh_wavelet+NM & 63.89 & $768 \times 1$ & Bray-Curtis & Walsh_wavelet_32X8+KMCG \\
Proposed VQ(CB size 16) & $\mathbf{7 4 \%}$ & $768 \times 1$ &
\end{tabular}

Observations: Accuracy increases from $52.67 \%$ to $74 \%$. Highest accuracy achieved with proposed technique applied on second method of Walsh wavelet.

Table 3. Variations in different factors considered for Classification.

\begin{tabular}{|c|c|c|c|}
\hline Sr. No. & Factors & No. of Variations & Variations \\
\hline 1 & Feature Vector size & 6 & $75 \times 1,150 \times 1,300 \times 1,450 \times 1,600 \times 1,768 \times 1$ \\
\hline 2 & Similarity Criteria & 4 & Euclidean, Manhattan, Cosine correlation, Bray-Curtis \\
\hline 3 & Code book size & 3 & $4,8,16$ \\
\hline 4 & Classifiers & 2 & NN \& NM Classifier \\
\hline 5 & VQ methods & 4 & LBG, KPE, KFCG, KMCG \\
\hline 6 & Walsh Wavelet matrix generation & 5 & $4 \times 64,8 \times 32,16 \times 16,32 \times 8,64 \times 4$ \\
\hline \multicolumn{4}{|c|}{$\begin{array}{l}\text { Observations: 'Walsh transform+row mean' method with factors } 1,2 \text { and } 4 \text { generates } 48 \text { results for single test image. Proposed VQ } \\
\text { technique over the 'Walsh transform+row mean' method with factors } 1,2,3 \text { and } 5 \text { generates } 288 \text { results for single test image. Walsh } \\
\text { wavelet method with factors } 1,2,4 \text { and } 6 \text { generates } 240 \text { results for single test image. Proposed VQ technique over the Walsh wavelet } \\
\text { method with factors } 1,2,3,5 \text { and } 6 \text { generates } 1440 \text { results for single test image. }\end{array}$} \\
\hline
\end{tabular}


Due to space constraint it is not possible to show the results of all the methods with all factor variation. But after implementing all the methods, it has been observed that proposed method of applying VQ over the earlier methods gives highest accuracy in most of the classes. In some classes like Bus, Dinosaur, Rose, Horse and Airplane, 'NM' method gives better results. It means that those classes are compact and they can be best represented by single feature vector. Bray-Curtis and Manhattan performance is almost similar and they both give better performance than other two similarity criteria in most classes. Euclidean and Cosine correlation shows similar performance. In sunset class and scooter class, cosine similarity gives better performance than any other distances.

Table 4. Confusion Matrix for Walsh_wavelet_32X8_NN method (Feature vector size: 768x1, Similarity measure: Manhattan distance, No. of training images: 55/class)

\begin{tabular}{|c|c|c|c|c|c|c|c|c|c|c|c|c|c|c|c|c|c|c|c|c|}
\hline & $\begin{array}{l}\overrightarrow{0} \\
.0 \\
.0 \\
=\end{array}$ & 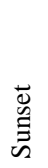 & 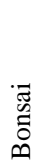 & 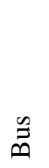 & 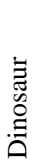 & $\frac{\vec{\Xi}}{\frac{\vec{U}}{0}}$ & 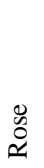 & $\begin{array}{l}\stackrel{D}{0} \\
\text { Oे } \\
\text { I }\end{array}$ & 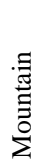 & తే & $\underset{\overparen{\Xi}}{\stackrel{\pi}{\Xi}}$ & 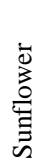 & 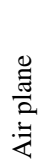 & ن & 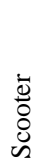 & 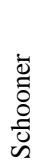 & 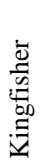 & $\begin{array}{l}\frac{5}{\sqrt{2}} \\
\text { 诖 } \\
\text { 离 }\end{array}$ & 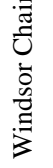 & 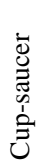 \\
\hline Ibis bird & 17 & 0 & 2 & 0 & 0 & 5 & 0 & 4 & 1 & 0 & 1 & 0 & 1 & 0 & 1 & 1 & 9 & 3 & 0 & 0 \\
\hline Sunset & 0 & 28 & 0 & 1 & 0 & 3 & 6 & 1 & 0 & 0 & 1 & 2 & 0 & 0 & 0 & 0 & 1 & 2 & 0 & 0 \\
\hline Bonsai & 1 & 0 & 26 & 1 & 0 & 2 & 0 & 2 & 0 & 0 & 2 & 0 & 0 & 3 & 1 & 0 & 3 & 1 & 1 & 2 \\
\hline Bus & 0 & 0 & 1 & 27 & 0 & 1 & 0 & 3 & 2 & 0 & 2 & 0 & 0 & 0 & 4 & 0 & 1 & 4 & 0 & 0 \\
\hline Dinosaur & 0 & 0 & 0 & 0 & 44 & 0 & 0 & 0 & 0 & 0 & 0 & 0 & 0 & 0 & 0 & 0 & 0 & 0 & 0 & 1 \\
\hline Elephant & 2 & 0 & 0 & 0 & 2 & 32 & 0 & 0 & 1 & 0 & 1 & 0 & 1 & 0 & 2 & 0 & 0 & 2 & 0 & 2 \\
\hline Rose & 0 & 0 & 0 & 0 & 0 & 0 & 41 & 1 & 0 & 0 & 2 & 0 & 0 & 0 & 0 & 0 & 0 & 1 & 0 & 0 \\
\hline Horse & 2 & 0 & 0 & 0 & 0 & 0 & 0 & 43 & 0 & 0 & 0 & 0 & 0 & 0 & 0 & 0 & 0 & 0 & 0 & 0 \\
\hline Mountain & 3 & 0 & 1 & 4 & 0 & 3 & 0 & 4 & 20 & 0 & 1 & 0 & 1 & 0 & 3 & 2 & 2 & 1 & 0 & 0 \\
\hline Car & 0 & 0 & 0 & 5 & 0 & 3 & 1 & 1 & 2 & 23 & 2 & 1 & 1 & 0 & 4 & 0 & 2 & 0 & 0 & 0 \\
\hline Panda & 1 & 0 & 0 & 1 & 0 & 0 & 0 & 3 & 1 & 2 & 36 & 0 & 0 & 0 & 1 & 0 & 0 & 0 & 0 & 0 \\
\hline Sunflower & 0 & 0 & 0 & 0 & 0 & 0 & 0 & 0 & 0 & 0 & 0 & 43 & 0 & 0 & 0 & 0 & 0 & 1 & 0 & 1 \\
\hline Air plane & 0 & 0 & 1 & 0 & 2 & 0 & 0 & 1 & 1 & 0 & 0 & 0 & 40 & 0 & 0 & 0 & 0 & 0 & 0 & 0 \\
\hline Coin & 1 & 0 & 0 & 0 & 0 & 2 & 0 & 0 & 0 & 0 & 0 & 0 & 0 & 40 & 0 & 0 & 1 & 1 & 0 & 0 \\
\hline Scooter & 0 & 0 & 1 & 1 & 0 & 0 & 0 & 0 & 1 & 0 & 0 & 0 & 0 & 0 & 41 & 0 & 0 & 1 & 0 & 0 \\
\hline Schooner & 0 & 0 & 0 & 0 & 0 & 0 & 0 & 0 & 0 & 0 & 1 & 0 & 0 & 0 & 0 & 44 & 0 & 0 & 0 & 0 \\
\hline Kingfisher & 0 & 0 & 0 & 0 & 0 & 2 & 3 & 4 & 0 & 0 & 4 & 0 & 0 & 0 & 0 & 0 & 27 & 3 & 0 & 2 \\
\hline Star Fish & 0 & 0 & 0 & 4 & 0 & 3 & 1 & 2 & 0 & 0 & 3 & 0 & 0 & 2 & 0 & 0 & 0 & 26 & 1 & 3 \\
\hline Windsor Chair & 2 & 0 & 0 & 0 & 3 & 0 & 0 & 0 & 0 & 0 & 1 & 0 & 0 & 0 & 0 & 0 & 0 & 2 & 37 & 0 \\
\hline Cup-saucer & 2 & 0 & 0 & 0 & 4 & 2 & 0 & 1 & 0 & 0 & 3 & 0 & 0 & 0 & 1 & 0 & 8 & 3 & 1 & 20 \\
\hline
\end{tabular}

Observations: Classes whose accuracy is more than 90\% are Dinosaur (98\%), Rose (91\%), Horse (96\%), Sunflower (96\%), Scooter(91\%) and Schooner (98\%). Worst performing classes are Ibis bird class with 38\% and cup-saucer class with 44\% accuracy. Few of Ibis bird images are misclassified as Kingfisher bird images due to similarity in structure and shape. Few of the cup-saucer class images are misclassified as Dinosaur class because of their plane background. Therefore, the accuracy of those classes degrades.

After Walsh transform applied to each column of an image, low frequency components get stored in the first few coefficients of each column. Hence maximum feature vector size is not required to get highest accuracy in first method. But in Walsh wavelet method, the low frequency components are spread. Hence, highest accuracy is obtained with feature vector of size 768x1.

\section{CONCLUSION}

The paper gives methods for generation of training set using Walsh Transform for classification of image database. If we increase training data, the accuracy of classification increases. In the paper, we have shown the results for 55\% of database (1100 images) for training, and tried to increase accuracy by implementing different techniques of feature vector generation. For classification, a simple and fast method, Nearest Neighbor classifier, is used. It has been observed that classes like dinosaur, rose give the better results with NM classifier than NN classifier. While the other classes give better performance with NN classifier. As we were analyzing this fact, it has been found that classes like dinosaur and rose are compact and close i.e. low intra-class distance and high inter-class distance compared to other training classes. After a lot of experimentation, we realized that instead of all training vectors per class or average training vector per class, there is a need to represent the class by few but more effective training feature vectors. It resulted in proposed method of applying vector quantization on 'Walsh transform+row mean+NN' method. This method increases accuracy from $61.22 \%$ to $63.22 \%$ and it has great advantage of reducing time required and storage space required for classification. However, in thirst of more accuracy, better feature vector representation is 
generated using wavelet transform. It has been observed that Walsh wavelet has increased an overall accuracy from $63.22 \%$ to $72.78 \%$. Manhattan and Bray-Curtis distance are more suitable similarity measures for this application. Walsh_wavelet_32x8_NN method gives highest accuracy of 72.78\%. In all VQ techniques it has been observed that KMCG with codebook size of 16 has given better performance. Hence, KMCG is applied on feature vectors generated by Walsh wavelet. The accuracy increased upto $74 \%$ for codebook size of 16 . Thus the proposed technique not only increases accuracy but also drastically reduces the size of training set (from 900 training vectors to 320 training vectors) results in less storage space required and faster classification.

\section{ACKNOWLEDGEMENTS}

We are extremely grateful to our research guide late Dr. H.B.Kekre for his valuable guidance, scholarly suggestions and consistent encouragement. Sir had always clarified our doubts despite his busy schedules and provided academic support and facilities to carry out this research work. We acknowledge our deep sense of gratitude towards him. Though he is not physically present with us, his work, words and blessings will remain with us forever.

\section{REFERENCES}

[1] Vailaya A., et al., "On Image Classification: City Images vs. Landscapes,” Pattern Recognition, Published by Elsevier Science Ltd., vol/issue: 31(12), pp. 1921-1935, 1998.

[2] Mio W., et al., “A learning approach to content-based image categorization and retrieval. VISAPP,” vol. 2, pp. 3643.

[3] N. Ghomsheh A and Talebpour A, "A New Method for Indoor-Outdoor Image Classification using Color Correlated Temperature,” International Journal of Image Processing (IJIP), vol/issue: 6(2), pp.167-181, 2012.

[4] Favorskaya M. and Proskurin A., "Image Categorization using Colour G-SURF Invariant to Ligyht Intensity," $19^{\text {th }}$ International Conference on Knowledge based and Intelligent Information and Engineering Systems. Procedia Computer Science, Elsevier publication, vol. 60, pp. 681-690, 2015.

[5] Gao S., et al., "Learning Category-Specific Dictionary and Shared Dictinary for Fine-Grained Image Categorization,” IEEE Transaction on Image Processing, vol/issue: 23(2), pp. 623-634, 2013.

[6] Bajwa I., et al., "Feature based Classification by using Principal Component Analysis," ICGST-GVIP journal, vol/issue: 9(2), pp. 11-17, 2009.

[7] Tax D., “One Class Classification: Concept Learning in the Absence of Counter Examples,” Ph.D. Thesis, Delft University of Technology, Delft, Netherland, 2001.

[8] Hamdi N, et al., "A New Approach based on Quantum Clustering and Wavelet Transform for Breast Cancer Classification: Comparative Study,” International Journal of Electrical and Computer Engineering (IJECE). Vol/issue: 5(5), 2015.

[9] Gopakumar C. and Rahuman R., "Randon Transform based Classification of Mammograms with improved Localization,” International Journal of Electrical and Computer Engineering (IJECE), vol/issue: 4(4), pp, 17-24, 2014.

[10] Le T., et al., “A New Support Vector Machine Method for Medical Image Classification,” IEEE 2nd European Workshop on Visual Information Processing (EUVIP), pp. 165-170, 2010.

[11] Olaode A., et al., "Unsupervised Classification of Images: A Review,” International Journal of Image Processing (IJIP), vol/issue: 8(5), pp. 325-342, 2014.

[12] Nazarloo M., et al., "Gender Classification using Hybrid of Gabor Filters and Binary Features of an Image," International Journal of Electrical and Computer Engineering (IJECE), vol/issue: 4(4), pp. 539-547, 2014.

[13] Jain A., et al., "Statistical pattern recognition: A review," IEEE transactions on Pattern Analysis and Machine Intelligence, vol/issue: 22(1), pp. 4-37, 2000.

[14] Song H., et al., "Adaptive Feature Selection and Extraction Approaches for Image Retrieval based on Region," Journal of Multimedia, vol/issue: 5(1), pp. 85-92, 2010.

[15] Haralick R., et al., "Textural Features for Image Classification,” IEEE Transactions on Systems, Man and Cybernetics, vol/issue: 3(6), pp. 610-621, 1973.

[16] Teague M., "Image Analysis via the general Theory of Moments," Journal of the Optical Society of America, vol. 70, pp. 920-930.

[17] Zhang D. and Lu G., "Shape-based Image Retrieval using generic Fourier Descriptor," Signal Processing: Image Communication, vol/issue: 17(10), pp. 825-848, 2002.

[18] Liping W. and Juncheng P., "Image Classification Algorithm based on Sparse Coding,” Journal of Multimedia, vol/issue: 9(1), pp. 114-122, 2014

[19] Boiman O., et al., "In Defense of Nearest-Neighbor Based Image Classification,” IEEE Conference on Computer Vision and Pattern Recognition (CVPR), 2008.

[20] Zhou S., et al., "Deep Adaptive Networks for Image Classification,” ICIMCS'10 Proceedings of the Second International conference on Internet Multimedia Computing and Service, pp. 61-64, 2010.

[21] Thai L., et al., "Image Classification using Support Vector Machine and Artificial Neural Network,” International Journal on Information Technology and Computer Science, MECS publication, pp. 32-38, 2012.

Walsh Transform Based Feature Vector Generation for Image Database Classification (Tanuja Sarode) 
[22] Walsh J., “A Closed set of Orthogonal Functions,” American Journal of Mathematics, vol. 45, pp. 5-24, 1923.

[23] Kekre H., et al., "DCT Applied to Row Mean and Column Vectors in Fingerprint Identification,” in Proceedings of Int. Conf. on Computer Networks and Security (ICCNS), 2008.

[24] Deza E. and Deza M., “Dictionary of Distances,” Elsevier, pp. 391, 2006.

[25] Kekre H. B., et al., "Walsh Transform over Row Mean and Column Mean using Image Fragmentation and Energy Compaction for Image Retrieval," International Journal on Computer Science and Engineering,(IJCSE), vol/issue: 2(1), pp. 47-54, 2010.

[26] Kekre H., et al., "Effect of Distance Measures on Transform based Image Classification,” International Journal of Engineering Science and Technology (IJEST), vol/issue: 4(8), pp. 3729-3742, 2012.

[27] Chen X. and Cham T., "Discriminative Distance Measures for Image Matching," International Conference on Pattern Recognition (ICPR), Cambridge, England, vol. 3, pp. 691-695, 2004.

[28] John P. Van De Geer, "Some Aspects of Minkowski distance,” Department of data theory, Leiden University. 1995.

[29] Kekre H., et al., "Inception of Hybrid Wavelet Transform using Two Orthogonal Transforms and It's use for Image Compression,” International Journal of Computer Science and Information Security, vol/issue: 9(6), pp. 80-87, 2011.

[30] Gray R., "Vector Quantization,” IEEE ASSP Mag, pp. 4-29, 1984.

[31] Linde Y., et al., “An Algorithm for Vector Quantizer Design,” IEEE Transactions on Communications, vol/issue: 28(1), pp. 84-95.

[32] Kekre H. and Sarode T., “Fast Improved Clustering Algorithms for Vector Quantization,” NCSPA, Padmashree Dr. D.Y.Patil Institute of Engineering and Technology, Pune, India, 2007.

[33] Kekre H. and Sarode T., "Fast Codebook Generation Algorithm for Color Images using Vector Quantization," International Journal of Computer Science and Information Technology, vol/issue: 1(1), pp. 7-12, 2009.

[34] Kekre H. and Sarode T., “An Efficient Fast Algorithm to generate Codebook for Vector Quantization,” First International conference on Emerging Trends in Engineering and Technology (ICETET), pp. 62-67, 2008.

[35] Wang J., et al., "SIMPLIcity: Semantics-sensitive Integrated Matching for Picture Libraries," IEEE Transaction on Pattern Analysis and Machine Intelligence, vol/issue: 23(9), pp. 947-963, 2001.

\section{BIOGRAPHIES OF AUTHORS}

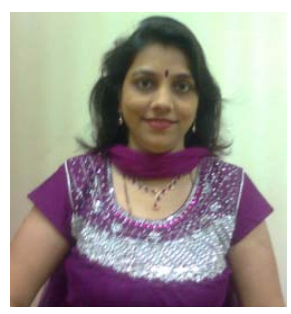

Dr.Tanuja K. Sarode has Received Bsc. (Mathematics) from Mumbai University in 1996, Bsc.Tech.(Computer Technology) from Mumbai University in 1999, M.E. (Computer Engineering) from Mumbai University in 2004 and Ph.D. from Mukesh Patel School of Technology, Management and Engineering, SVKM's NMIMS University, Vile-Parle (W), Mumbai, INDIA. She has more than 10 years of experience in teaching. Currently working as Associate Professor in Dept. of Computer Engineering at Thadomal Shahani Engineering College, Mumbai. She is life member of IETE, ISTE, member of International Association of Engineers (IAENG) and International Association of Computer Science and Information Technology (IACSIT), Singapore. Her areas of interest are Image Processing, Signal Processing and Computer Graphics. She has more than 100 papers in National /International Conferences/journal to her credit.

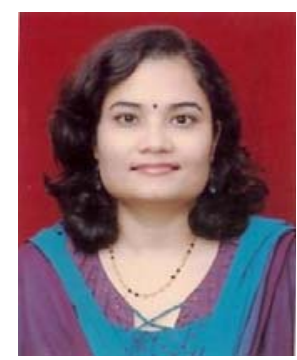

Jagruti K. Save has received B.E. (Computer Engg.) from Mumbai University in 1996, M.E. (Computer Engineering) from Mumbai University in 2004, currently Pursuing Ph.D. from Mukesh Patel School of Technology, Management and Engineering, SVKM's NMIMS University, Vile-Parle (W), Mumbai, INDIA. She has more than 10 years of experience in teaching. Currently working as Associate Professor in Dept. of Information Technology at Fr. Conceicao Rodrigues College of Engg., Bandra, Mumbai. Her areas of interest are Image Processing, Neural Networks, Fuzzy systems, Data base management and Computer Vision. She has 10 papers in National /International Conferences/journal to her credit. 